\title{
Predictive factors of the treatment outcome in patients with advanced biliary tract cancer receiving gemcitabine plus cisplatin as first-line chemotherapy
}

\author{
Yuko Suzuki $^{1} \cdot$ Motoyasu Kan $^{1} \cdot$ Gen Kimura $^{1} \cdot$ Kumiko Umemoto $^{1}$ • \\ Kazuo Watanabe $^{1}$ Mitsuhito Sasaki ${ }^{1} \cdot$ Hideaki Takahashi ${ }^{1} \cdot$ Yusuke Hashimoto $^{1}$. \\ Hiroshi Imaoka $^{1} \cdot$ Izumi Ohno $^{1} \cdot$ Shuichi Mitsunaga $^{1} \cdot$ Masafumi Ikeda $^{1}(\mathbb{C}$
}

Received: 10 August 2018/Accepted: 1 October 2018/Published online: 8 October 2018

(C) The Author(s) 2018

\begin{abstract}
Background Few studies have clearly identified the prognostic factors in patients with advanced biliary tract cancer (BTC) receiving gemcitabine plus cisplatin (GC) which is acknowledged as standard chemotherapy regimen.

Objectives The aim of this study was to identify predictive factors of the overall survival (OS) in advanced BTC patients receiving GC therapy.

Methods Data of 307 patients with advanced BTC who received GC therapy as the first-line chemotherapy at our institution from January 2007 to June 2017 were reviewed retrospectively. The patients were randomly assigned to the investigation or the validation dataset at the ratio of $2: 1$. Multivariate analysis was conducted to identify the prognostic factors, a prognostic index is proposed from the investigation dataset, and the usefulness of this index was confirmed in the validation dataset.

Results Multivariate analysis identified poor performance status, elevated serum lactate dehydrogenase, and elevated neutrophil-to-lymphocyte ratio as independent unfavorable predictors. The patients could be classified into three groups according to these factors, and it was found that the outcomes differed significantly among the three groups ( $P=0.0002$, good- vs. intermediate-prognosis groups; $P=0.005$, intermediate- vs. poor-prognosis groups). When this index was applied to the validation dataset, the OS was confirmed to differ significantly among the three groups
\end{abstract}

Masafumi Ikeda

masikeda@east.ncc.go.jp

1 Department of Hepatobiliary and Pancreatic Oncology, National Cancer Center Hospital East, 6-5-1 Kashiwanoha, Kashiwa 277-8577, Japan
$(P=0.04$, good- vs. intermediate-prognosis groups, $P<0.0001$, intermediate- vs. poor-prognosis groups).

Conclusions We identified three predictors of the OS in patients with advanced BTC receiving GC therapy in this study, based on which we could classify the patients into three risk groups.

Keywords Biliary tract cancer - Prognostic factor . Validation · Chemotherapy - Gemcitabine and cisplatin

\section{Introduction}

Biliary tract cancer (BTC), while being an uncommon malignancy in Western countries, is relatively common, accounting for $2-3 \%$ of all malignant neoplasms, in Japan; approximately 22,000 new patients are registered and 18,000 die of the disease each year in Japan [1]. BTC has a dismal prognosis and surgical resection is the only treatment modality that offers any chance of cure. However, in many patients, the diagnosis is made only at an advanced stage of the disease, by which time, surgical resection is no longer applicable. On the other hand, even in patients undergoing curative resection, recurrence occurs at a very high rate [2-4]. Systemic chemotherapy plays an important role in the treatment of unresectable or recurrent BTC. In a phase III trial (ABC-02) conducted in the United Kingdom (UK), gemcitabine plus cisplatin (GC) therapy improved the survival outcome (median overall survival [OS], 11.7 months) as compared to treatment with gemcitabine alone [median OS, 8.1 months, hazard ratio, 0.64; 95\% confidence interval (CI) $0.52-0.80 ; P<0.0001$ ] [5]. In a randomized phase II trial (the BT22 trial) conducted in Japan at around the same time, a similar efficacy and safety 
of GC therapy were observed (median OS: GC, 11.2 months; gemcitabine alone, 7.7 months; hazard ratio, $0.69 ; 95 \%$ CI $0.42-0.80 ; P<0.0001$ ) [6]. Based on the above-described results, GC is now established as the standard first-line chemotherapy for advanced BTC.

Subsequently, a randomized phase III trial (JCOG1113) conducted in Japan showed the non-inferiority of the combined gemcitabine plus S-1 (GS) therapy to GC therapy in terms of the overall survival outcome [7]. Therefore, GS is also currently available as one of the chemotherapy options for patients with advanced BTC, although GC still remains the standard first-line regimen. It is important to precisely identify patients who can derive survival benefit from GC therapy. However, from the few studies conducted until date, no predictors of the OS have been identified yet. In addition, few prognostic indexes have been constructed and few validation studies of the prognostic index have been conducted [8-12]. The purpose of this study, therefore, was to identify and then conduct the prognostic index of OS, and validate it, in patients with advanced BTC receiving GC as first-line chemotherapy.

\section{Materials and methods}

\section{Patients}

This study included a total of 307 patients with histologically or cytologically proven advanced BTC, extrahepatic cholangiocarcinoma, intrahepatic cholangiocarcinoma, gallbladder or ampullary carcinoma, who were started on GC as first-line chemotherapy at the National Cancer Center Hospital East, Kashiwa, Japan, between January 2007 and June 2017. All clinical data were reviewed retrospectively from the hospital records. The database was fixed for analysis in December 2017. All patients were randomly assigned to the investigation dataset (205 patients) or the validation dataset (102 patients) at the ratio of 2:1. This retrospective study was conducted in accordance with the 1964 Declaration of Helsinki and its later amendments, and the protocol was approved by the Ethics Committee of National Cancer Center Hospital East (Approval no. 2017-322).

\section{Treatment}

All patients received GC therapy: cisplatin $\left(25 \mathrm{mg} / \mathrm{m}^{2}\right)$, followed by gemcitabine $\left(1000 \mathrm{mg} / \mathrm{m}^{2}\right)$ administered by intravenous infusion on days 1 and 8 of each 3 -week cycle. A total of 16 doses of cisplatin was administered $(400 \mathrm{mg} /$ $\mathrm{m}^{2}$ ), unless there was evidence of disease progression or unacceptable toxicity, while gemcitabine alone was continued indefinitely until evidence of disease progression or appearance of unacceptable toxicity.

\section{Assessment of toxicity and efficacy}

All patients underwent physical examination and assessments for evidence of drug toxicity before and every 1 or 2 weeks after the initiation of GC therapy. Toxicities appearing during GC therapy were graded according to the Common Terminology Criteria for Adverse Events (CTCAE) version 4.0. Computed tomography (CT) or magnetic resonance imaging (MRI) was performed every 4-8 weeks, the tumor responses were assessed on the images by both medical oncologists and radiologists, in accordance with the Response Evaluation Criteria In Solid Tumors (RECIST) version 1.1, and the best response in each patient was recorded.

\section{Statistical analysis}

OS was calculated as the time interval from the date of initiation of the GC therapy until the date of death. Progression-free survival (PFS) was calculated from the date of initiation of the GC therapy until the date of documentation of disease progression or death. Patients who did not show disease progression and patients who died were excluded at the date of their last follow-up visit or the date of their death. Univariate analysis was performed using Mann-Whitney $U$ test for continuous variables and Chisquared test for categorical variables. The Kaplan-Meier method was used to estimate the time-to-event distribution, and $P$ values were calculated using a log-rank test. Hazard ratios were calculated using Cox proportional hazard model. Age, body mass index, maximum tumor size and laboratory parameters were set as continuous variables, while other factors were used as categorical variables. Statistically significant variables $(P<0.05)$ identified by univariate analysis were entered into the multivariate analysis model. After identification of the prognostic factors by multivariate analysis, the continuous variables were also divided into two categories, followed by receiver operating characteristics (ROC) curve analysis to construct a prognostic index. ROC curve was used to determine the optimal cutoff value that predicted the survival and maximized both the sensitivity and the specificity of continuous variables. The prognostic index was calculated based on the statistically significant prognostic factors identified by multivariate analysis. All tests were two sided and $P<0.05$ was considered as denoting statistical significance. All the statistical analyses were performed using the JMP 13.0 software for Macintosh, version 13.2 (SAS Institute Inc., Cary, North Carolina, USA). 


\section{Results}

\section{Patient characteristics}

A total of 307 patients with advanced BTC received GC as the first-line chemotherapy at our institution between January 2007 and June 2017. By the time of the analysis, 226 had already died. The baseline characteristics and clinical data of all the patients $(n=307)$ included in the investigation dataset $(n=205)$ and validation dataset $(n=102)$ are summarized in Table 1. Although there were a few missing data on the patient characteristics (serum CEA level data missing in 2 patients, and serum CA19-9 level data missing in 3 patients), there were no significant differences in the patient characteristics between the investigation and validation datasets.

\section{Efficacy of GC}

The objective tumor response could be assessed by CT/ MRI in a total of 304 patients, in accordance with RECIST version 1.1 (Table 2). Complete response (CR), partial response (PR), and stable disease (SD) were observed in 9 (2.9\%), 43 (14.0\%), and 196 (63.9\%) patients, respectively, representing an overall response rate $(\mathrm{CR}+\mathrm{PR})$ of $16.9 \%$ and tumor control rate $(\mathrm{CR}+\mathrm{PR}+\mathrm{SD})$ of $80.8 \%$. Moreover, two patients each who showed CR and PR underwent curative resection after the GC therapy. Figure 1 shows the Kaplan-Meier curves for OS (Fig. 1a) and PFS (Fig. 1b) in the entire study population of 307 patients that had received GC therapy. The median OS, PFS and 1-year survival rate were 13.0 months [95\% confidence interval (CI) 11.0-13.9], 6.9 months (95\% CI 5.9-7.7), and $52.7 \%$, respectively.

There were no significant differences in the efficacy of GC therapy between the investigation and validation datasets (Table 2).

\section{Treatment and toxicity}

The median duration of treatment was 6.0 months (range 0.03-47.7 months). The GC therapy had to be discontinued in 292 patients $(95.1 \%)$, the main reasons for the treatment discontinuation being disease progression (247 patients, $80.5 \%$ ) and treatment-related toxicities (17 patients, $5.5 \%$ ). A total of 181 patients $(58.9 \%)$ received subsequent therapies after failure of GC therapy. In most of these cases ( $n=152,84.0 \%$ ), the second-line treatment was S-1 monotherapy. The toxicity of GC therapy is summarized in Table 3. The mainly encountered grade 3 or more severe toxicities were neutropenia (172 patients, 56.0\%), anemia (102 patients, 33.2\%), leukopenia (101 patients, 32.9\%), lymphocytes decreased (70 patients, 22.8\%), thrombocytopenia (41 patients, $13.3 \%$ ), alkaline phosphatase elevated (38 patients, $12.4 \%$ ), and aspartate aminotransferase elevated (37 patients, $12.1 \%$ ); there were no treatment-related deaths. Dose modifications of gemcitabine and cisplatin were needed in $40.7 \%$ and $29.3 \%$ of all patients, respectively.

\section{Identification of predictive factors of the survival outcome and construction of a prognostic index from the investigation dataset}

The results of univariate analysis of a total of 22 variables in the investigation dataset are shown in Table 4. Among these variables, a total of 13 factors recorded before the start of GC therapy were associated with a worse OS. Multivariate analysis identified poor performance status (PS), increased serum lactate dehydrogenase (LDH) level, and elevated neutrophil-to-lymphocyte ratio (NLR) as independent predictors of a worse OS (Table 4).

For clinical application, we constructed a prognostic index based on the three prognostic factors identified by multivariate analysis, namely PS, serum LDH level and the NLR. Then, on the basis of this index, the patients could be classified into three risk groups. Because the hazard ratios calculated by multivariate analysis after categorizing the serum LDH and NLR into two variables (data not shown) did not differ among the three identified prognostic factors, we calculated the prognostic index on the basis of the number of independent prognostic factors. The cutoff serum LDH level was determined as upper limit of normal (245 IU/L) because the cutoff value calculated from the ROC curve was approximately equivalent to the upper limit of normal. The cutoff value for the NLR was determined to be 3.0 , because the cutoff value calculated from the ROC curve was almost equivalent to that determined in previous investigations [8, 13-15]. Then the patients were divided into three groups, as follows: the good-prognosis group [none of the poor prognostic factors present, 72 patients $(35.1 \%)$ ], intermediate- prognosis group [1 or 2 poor prognostic factors present, 114 patients $(55.6 \%)]$, and poor-prognosis group [all three poor prognostic factors present, 19 patients $(9.3 \%)]$. The median OS times in the good-, intermediate-, and poor-prognosis groups were 16.3 months (95\% CI 13.2-23.1), 11.3 months (95\% CI 9.2-13.4), and 5.3 months (95\% CI 3.0-7.1), respectively. Thus, the outcomes were found to differ significantly among the three groups: good- vs. intermediate-prognosis 
Table 1 Baseline patient characteristics

\begin{tabular}{|c|c|c|c|c|}
\hline Variable & Total $(n=307)$ & Investigation dataset $(n=205)$ & Validation dataset $(n=102)$ & $P$ value* \\
\hline \multicolumn{5}{|l|}{ Age, years } \\
\hline Median [range] & $68[33-85]$ & $68[35-83]$ & $68[33-85]$ & 0.81 \\
\hline \multicolumn{5}{|l|}{ Gender, $n(\%)$} \\
\hline Male & $179(58.3)$ & $116(56.6)$ & $63(61.8)$ & \multirow[t]{2}{*}{0.38} \\
\hline Female & $128(41.7)$ & $89(43.4)$ & $39(38.2)$ & \\
\hline \multicolumn{5}{|l|}{ ECOG PS, n (\%) } \\
\hline 0 & $199(64.8)$ & $130(63.4)$ & $69(67.7)$ & \multirow[t]{2}{*}{0.46} \\
\hline $1-2$ & $108(35.2)$ & $75(36.6)$ & $33(32.3)$ & \\
\hline \multicolumn{5}{|l|}{ Primary tumor site, $n(\%)$} \\
\hline Intrahepatic bile duct & 103 (33.6) & $66(32.2)$ & $37(36.3)$ & \multirow[t]{4}{*}{0.53} \\
\hline Extrahepatic bile duct & $84(27.3)$ & $55(26.8)$ & $29(28.4)$ & \\
\hline Gallbladder & $108(35.2)$ & $74(36.1)$ & $34(33.3)$ & \\
\hline Ampulla of Vater & $12(3.9)$ & $10(4.9)$ & $2(2.0)$ & \\
\hline Maximum tumor size, mm, median [range] & $40[5-165]$ & $39[9-165]$ & $36[5-140]$ & 0.54 \\
\hline \multicolumn{5}{|l|}{ Extent of disease, $n(\%)$} \\
\hline Locally advanced & $62(20.2)$ & $41(20.0)$ & $21(20.6)$ & \multirow[t]{2}{*}{0.90} \\
\hline Metastatic & $245(79.8)$ & $164(80.0)$ & $81(79.4)$ & \\
\hline \multicolumn{5}{|l|}{ Metastatic site, $n(\%)$} \\
\hline Liver & $80(26.1)$ & $55(26.8)$ & $25(24.5)$ & 0.66 \\
\hline Lung & $35(11.4)$ & $25(12.2)$ & $10(9.8)$ & 0.53 \\
\hline Lymph node & $179(58.3)$ & $122(59.5)$ & $57(55.9)$ & 0.54 \\
\hline Peritoneum & $85(27.8)$ & $52(25.4)$ & $33(32.4)$ & 0.20 \\
\hline \multicolumn{5}{|l|}{ Type of tumor, $n(\%)$} \\
\hline Adenocarcinoma & $293(95.4)$ & 194 (94.6) & $99(97.1)$ & \multirow[t]{3}{*}{0.44} \\
\hline Adenosquamous carcinoma & $3(1.0)$ & $2(1.0)$ & $1(1.0)$ & \\
\hline Others $^{\mathrm{a}}$ & $11(3.6)$ & $9(4.4)$ & $2(1.9)$ & \\
\hline Prior surgical resection, $n(\%)$ & $72(23.5)$ & $48(23.4)$ & $24(23.5)$ & 0.98 \\
\hline Biliary drainage, $n(\%)$ & $135(43.9)$ & $90(43.9)$ & $45(44.1)$ & 0.97 \\
\hline Subsequent chemotherapy, $n(\%)$ & $181(58.9)$ & $120(58.5)$ & $61(59.8)$ & 0.83 \\
\hline \multicolumn{5}{|l|}{ Blood examinations, median [range] } \\
\hline White blood cell count $/ \mu \mathrm{L}$ & $6000[2100-26,100]$ & $5900[2100-26,100]$ & $6100[2500-25,900]$ & 0.94 \\
\hline Hemoglobin, g/dL & $12.0[7.3-19.0]$ & $12.0[7.3-19.0]$ & $12.0[7.9-15.1]$ & 0.93 \\
\hline Platelets, $/ \mu \mathrm{L}$ & $20.9[7.9-67.4]$ & $20.5[8.0-44.3]$ & $21.8[7.9-67.4]$ & 0.44 \\
\hline Albumin, $\mathrm{g} / \mathrm{dL}$ & $3.8[2.0-5.2]$ & $3.8[2.2-5.2]$ & $3.7[2.0-4.7]$ & 0.42 \\
\hline Total bilirubin, $\mathrm{mg} / \mathrm{dL}$ & $0.7[0.2-3.0]$ & $0.7[0.2-3.0]$ & $0.8[0.3-2.8]$ & 0.96 \\
\hline ALP, IU/L & $443[75-3395]$ & $429[158-3395]$ & $501[75-3056]$ & 0.24 \\
\hline LDH, IU/L & $180[85-1211]$ & $180[85-1211]$ & 179 [111-973] & 0.94 \\
\hline $\mathrm{CRP}, \mathrm{mg} / \mathrm{dL}$ & $0.65[0.03-18.35]$ & & & \\
\hline NLR & $2.99[0.51-25.9]$ & $2.91[0.51-17.4]$ & $3.11[1.06-25.9]$ & 0.77 \\
\hline \multicolumn{5}{|l|}{ Tumor marker, median [range] } \\
\hline $\mathrm{CEA}, \mathrm{ng} / \mathrm{mL}$ & $3.8[0.2-1482]$ & $3.8[0.2-1482]$ & $4.0[0.3-606]$ & 0.57 \\
\hline CA19-9, U/mL & $168[0.1-129,820]$ & $155[0.1-129,820]$ & $209[0.1-68,120]$ & 0.78 \\
\hline
\end{tabular}

ECOG PS Eastern Cooperative Oncology Group Performance Status, ALP alkaline phosphatase, $L D H$ lactate dehydrogenase, $C R P$ C-reactive protein, NLR neutrophil-to-lymphocyte ratio, CEA carcinoembryonic antigen, CA19-9 carbohydrate antigen 19-9

*The difference between the investigation dataset and validation dataset

${ }^{a}$ These patients were diagnosed by cytology class $\mathrm{V}$ with imaging findings 
Table 2 Best tumor response according to RECIST version 1.0

\begin{tabular}{lccc}
\hline & Total, $n(\%)$ & Investigation dataset $(n=205)$ & Validation dataset $(n=102)$ \\
\hline Best response & & & \\
CR & $9(2.9)$ & $8(3.9)$ & $1(1.0)$ \\
PR & $43(14.0)$ & $30(14.6)$ & $13(12.8)$ \\
SD & $196(63.9)$ & $126(61.5)$ & $70(68.6)$ \\
PD & $56(18.2)$ & $39(19.0)$ & $17(16.7)$ \\
NE & $3(1.0)$ & $2(1.0)$ & $1(1.0)$ \\
ORR (CR + PR) & $52(16.9)$ & $38(18.5)$ & $14(13.7)$ \\
DCR (CR + PR + SD) & $248(80.8)$ & $164(80.0)$ & $84(82.4)$ \\
\hline
\end{tabular}

$C R$ complete response, $P R$ partial response, $S D$ stable disease, $P D$ progressive disease, $N E$ not evaluable, $O R R$ overall response rate, $D C R$ disease control rate

*The difference between the investigation dataset and validation dataset

groups, $P=0.0002$ and intermediate- vs. poor-prognosis groups, $P=0.005$ (Fig. 2a).

\section{Validation of the prognostic index in the validation set}

The median OS, PFS and 1-year survival rate in the validation dataset were 13.2 months (95\% CI 10.0-15.5), 5.9 months (95\% CI 4.5-7.2), and $51.8 \%$, respectively. The prognostic index constructed from the investigation dataset was applied to the validation dataset. In the validation dataset, the median OS in the good-, intermediate-, and poor-prognosis groups were 15.8 months (95\% CI 11.5-20.5), 10.8 months $(95 \%$ CI $8.9-14.4)$, and 3.6 months (95\% CI 2.1-7.0), respectively (Fig. 2b). The differences among the three groups were statistically significant: good- vs. intermediate-prognosis group, $P=0.04$, and intermediate- vs. poor-prognosis groups, $P<0.0001$.

\section{Discussion}

In patients with advanced BTC, systemic chemotherapy is one of the important treatment modalities to improve survival. While some investigators have reported on the prognostic factors in advanced BTC patients receiving GC therapy, these prognostic factors had not yet been confirmed as valid, because of the limited number of patients enrolled. The present study was aimed at evaluating the efficacy and safety of GC therapy, and identifying the predictive factors of the OS in a relatively large number of the patients, that is, over 300 patients, with advanced BTC receiving GC therapy. The efficacy and safety parameters of GC therapy at our institution were almost similar to those reported from the phase III trial of GC therapy (the ABC-02 trial) conducted in the UK. In our patient cohort, we identified three factors (poor PS, increased serum LDH level and elevated NLR) as independent predictors of an unfavorable OS from among 13 potential factors by multivariate analysis of the data from the 205 patients included in the investigation dataset. We constructed a prognostic index for clinical application based on these three independent prognostic factors, and then confirmed the usefulness of this prognostic index in the 102 patients of the validation dataset.

PS was identified as one of the most robust and important prognostic factors in patients with various advanced cancer. Although PS is a somewhat subjective and vague assessment of the physical condition of cancer patients, it was identified as the most important predictor of the survival in advanced BTC patients receiving chemotherapy. Indeed, some previous studies have also reported PS as a prognostic factor in patients with advanced BTC, in conformity with our findings [8-11, 13, 14, 16-21]. Although previous reports suggest a poor prognosis in patients with PS $2[8,10,11,17,18,21]$, both patients with PS 2 and PS 1 had a poor prognosis in this study. The median OS in the patients with PS 0, PS 1 and PS 2 in this study was 13.9, 9.0 and 5.4 months, respectively. While the difference in the OS between the PS 0 and PS 1 patients was significant $(P<0.0001)$, that between the patients with PS 1 and PS 2 was not significant $(P=0.16)$. Therefore, we divided the cohort into patients with PS 0 and PS 1-2 for predicting the prognosis. Our results were consistent with those described in some other reports $[9,16,19,20]$. 
Fig. 1 Kaplan-Meier curves of OS (a) and PFS (b) in patients with advanced BTC receiving gemcitabine plus cisplatin (GC) therapy. The median OS and PFS were 13.0 months $(95 \%$ CI 11.0-13.9) and 6.9 months (95\% CI 5.9-7.7), respectively. $O S$ overall survival, $P F S$ progression-free survival, $C I$ confidence interval
LDH is a glycolytic enzyme with a key role in the conversion of pyruvate to lactate under anaerobic conditions. In hypoxic environments, as in tumor tissues, hypoxia-inducible factor $1 \alpha$ (HIF-1 $1 \alpha$ ) is commonly induced, which activates both LDH-A, which is one of the LDH isozymes, and pro-angiogenesis factors such as vascular endothelial growth factor (VEGFA, VEGFR) via the
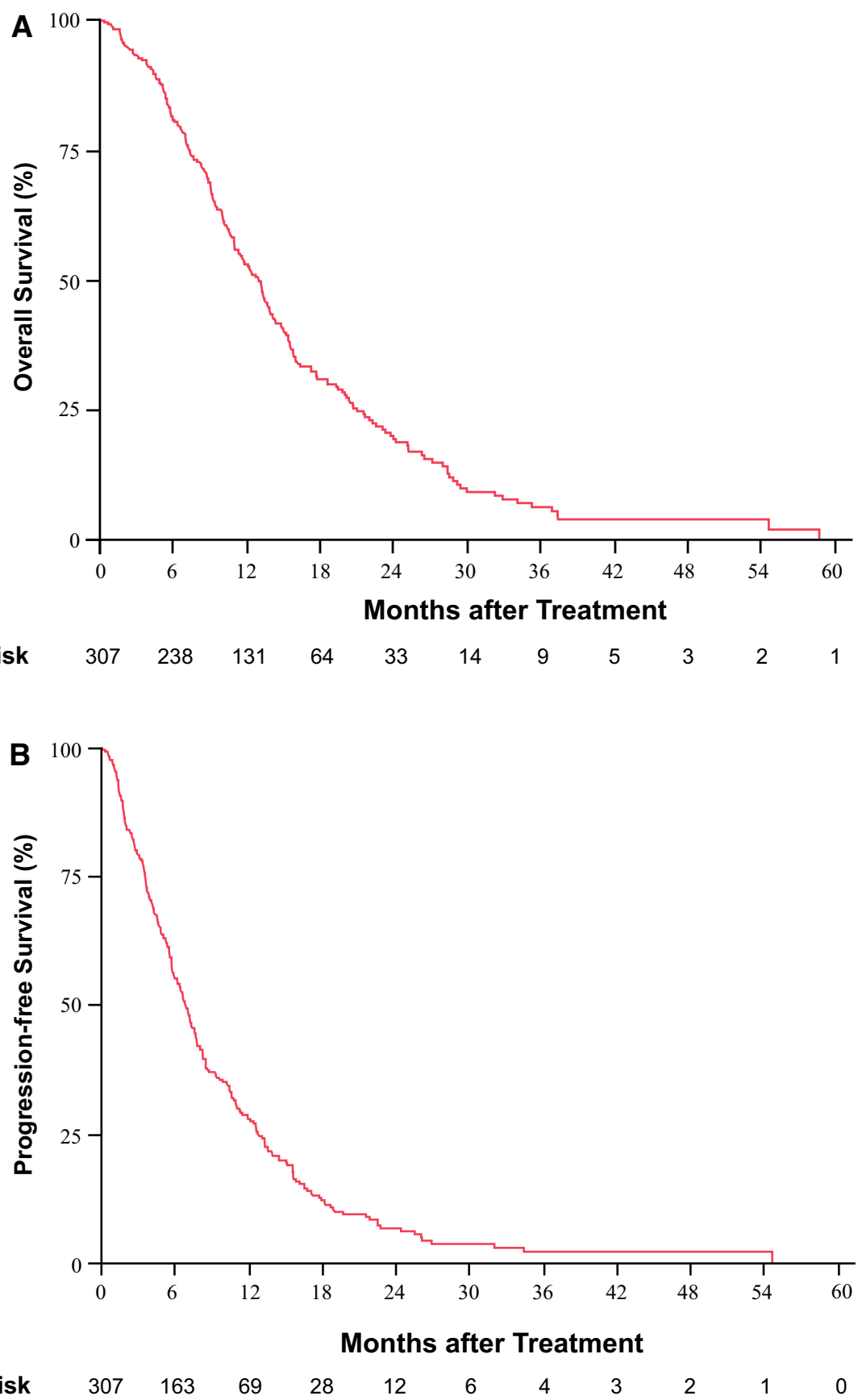
Table 3 Treatment-related toxicity graded according to CTCAE version 4.0

\begin{tabular}{|c|c|c|c|}
\hline \multirow[t]{2}{*}{ Variable } & \multicolumn{3}{|c|}{ Toxicity grade, $n(\%)$} \\
\hline & Grade 3 & Grade 4 & Any grades \\
\hline \multicolumn{4}{|l|}{ Hematological } \\
\hline Leucopenia & $97(31.6)$ & $4(1.3)$ & $283(92.2)$ \\
\hline Anemia & $93(30.3)$ & $9(2.9)$ & $304(99.0)$ \\
\hline Thrombocytopenia & $28(9.1)$ & $13(4.2)$ & $223(72.6)$ \\
\hline Neutropenia & $127(41.4)$ & $45(14.7)$ & $267(87.0)$ \\
\hline Lymphocyte decreased & $63(20.5)$ & $7(2.3)$ & $223(72.6)$ \\
\hline \multicolumn{4}{|l|}{ Non-hematological } \\
\hline Fatigue & $3(1.0)$ & $0(0)$ & $172(56.0)$ \\
\hline Anorexia & $5(1.6)$ & $0(0)$ & $141(45.9)$ \\
\hline Nausea & $1(0.3)$ & $0(0)$ & $101(32.9)$ \\
\hline Vomiting & $1(0.3)$ & $0(0)$ & $16(5.2)$ \\
\hline Febrile neutropenia & $5(1.6)$ & $0(0)$ & $5(1.6)$ \\
\hline Alopecia & $0(0)$ & $0(0)$ & $29(9.4)$ \\
\hline Neuropathy & $2(0.7)$ & $0(0)$ & $42(13.4)$ \\
\hline Thrombosis & $4(1.3)$ & $2(0.7)$ & $11(3.6)$ \\
\hline AST elevated & $37(12.1)$ & $0(0)$ & $213(69.4)$ \\
\hline ALT elevated & $34(11.1)$ & $0(0)$ & $201(65.5)$ \\
\hline ALP elevated & $38(12.4)$ & $0(0)$ & $258(84.0)$ \\
\hline Creatinine increased & $5(1.6)$ & $0(0)$ & $137(44.6)$ \\
\hline Albumin decreased & $15(4.9)$ & $0(0)$ & $265(86.3)$ \\
\hline Hyponatremia & $26(8.5)$ & $0(0)$ & $188(61.2)$ \\
\hline
\end{tabular}

AST aspartate aminotransferase, $A L T$ alanine aminotransferase, $A L P$ alkaline phosphatase

reports to the previous studies $[12,18,19,30]$, our study also suggested that elevated serum LDH might be associated with a worse OS in patients with advanced BTC receiving GC therapy.

Elevated NLR had been recognized as an indicator of a poor prognosis [31, 32] and poor tumor response [33, 34] in many cancers. Several investigators have reported the value of NLR as a predictor of the OS [8, 13, 14, 35, 36]. An elevated NLR might represent induced immunocompetence or neutrophilia. It is well known that neutrophilia contributes to stimulating the tumor microenvironment, specifically promoting cell proliferation, angiogenesis, invasion and metastasis in cancer $[14,35,37,38]$. Neutrophilia also plays a role in inhibiting the immune system by suppressing the cytolytic activity of immune cells such as lymphocytes, $T$ cells and natural killer cells [39]. On the other hand, lymphocytes are known as indispensable mediators in the anti-tumor immune system. Some previous studies have revealed that a decreased count of lym- phocytes in a tumor is associated with a worse response to chemotherapy and a poor prognosis in cancer patients [32, 40-42]. Therefore, an elevated NLR might be associated with potential tumor growth.

Using the three aforementioned prognostic factors, we constructed a prognostic index, based on which we could divide our patients into three different prognosis groups, and the usefulness of this index was confirmed in the validation dataset. This index is simple and easy to apply for the prediction of the prognosis prior to the initiation of chemotherapy in the daily clinical setting. The OS in the poor-prognosis group, with all three poor prognostic factors, was extremely dismal (median OS, 3.6-5.3 months). Therefore, it would be desirable for such patients to be offered the best supportive care or a more conservative regimen. This index may be helpful in predicting the life expectancy in advanced BTC patients receiving GC therapy and be useful to stratify patients in future clinical trials.

In this study, nine patients showed CR, and five of these patients with $\mathrm{CR}$ were still alive at the time of the analysis. One of these patients, and also one patient who showed PR, underwent conversion surgery after the GC therapy. Although GC therapy generally serves as palliative chemotherapy in patients with advanced BTC, it may have a potential role in enabling conversion surgery.

There were three major limitations of this study. First, as this study was conducted retrospectively, we could not include any pre-treatment data, such as weight loss, intensity of pain, or quality of life, which were not fully documented in the hospital records. Second, external validation could not have performed to allow generalizability of our findings, because this was a single-institution study. Third, these predictive factors were not specific to advanced BTC. Although we included some specific factors in patients with advanced BTC in the analysis as potential predictive factors, such as the serum total bilirubin level, biliary drainage, tumor size, serum CA19-9 level and presence/absence of metastatic disease, none of these factors was identified as a predictor of the OS in our cohort. Further investigation to identify other novel biliary cancerspecific markers is needed. In contrast, the strength of this study was the large sample size with few missing patient data recruited from a major Japanese cancer center. In addition, the patient selections, management of GC therapy, and assessment of the tumor response were unified as this was a single-institution study. Therefore, because of the solid and similar patient data, the efficacy and safety of GC therapy in our cohort were comparable to that reported from the $\mathrm{ABC}-02$ and $\mathrm{BT}-22$ trials. 
Table 4 Univariate and multivariate analyses using a Cox proportional hazard model to identify predictors of the overall survival

\begin{tabular}{|c|c|c|c|c|c|c|c|}
\hline \multirow[t]{2}{*}{ Variable } & \multicolumn{4}{|c|}{ Univariate analysis } & \multicolumn{3}{|c|}{ Multivariate analysis } \\
\hline & OS, months ${ }^{\mathrm{a}}$ & HR & $95 \% \mathrm{CI}$ & $P$ value & HR & $95 \% \mathrm{CI}$ & $P$ value \\
\hline Age & & 1.010 & $0.991-1.031$ & 0.30 & & & \\
\hline \multicolumn{8}{|l|}{ Gender } \\
\hline Male & & 1.000 & & & & & \\
\hline Female & 12.4 & 1.110 & $0.799-1.536$ & 0.53 & & & \\
\hline Body Mass Index & 13.2 & 0.992 & $0.936-1.050$ & 0.78 & & & \\
\hline \multicolumn{8}{|l|}{ ECOG PS } \\
\hline 0 & 13.9 & 1.000 & & & 1.000 & & \\
\hline $1-2$ & 7.6 & 2.383 & $1.699-3.327$ & $<0.001$ & 1.709 & $1.162-2.497$ & $<0.001$ \\
\hline \multicolumn{8}{|l|}{ Primary tumor site } \\
\hline Bile duct ${ }^{\mathrm{b}}$ & 13.2 & 1.000 & & & & & \\
\hline Gallbladder & 11.3 & 1.235 & $0.877-1.721$ & 0.22 & & & \\
\hline Maximum tumor size & & 1.007 & $1.003-1.012$ & 0.002 & 0.995 & $0.988-1.0008$ & 0.092 \\
\hline \multicolumn{8}{|l|}{ Extent of disease } \\
\hline Locally advanced & 18.6 & 1.000 & & & 1.000 & & \\
\hline Metastatic & 11.6 & 1.509 & $1.023-2.298$ & 0.038 & 1.579 & $1.163-2.493$ & 0.067 \\
\hline \multicolumn{8}{|l|}{ Liver metastasis } \\
\hline Absent & 13.3 & 1.000 & & & & & \\
\hline Present & 10.2 & 1.424 & $0.979-2.034$ & 0.064 & & & \\
\hline \multicolumn{8}{|l|}{ Peritoneal dissemination } \\
\hline Absent & 13.6 & 1.000 & & & 1.000 & & \\
\hline Present & 8.8 & 1.559 & $1.074-2.219$ & 0.020 & 1.218 & $0.805-1.816$ & 0.35 \\
\hline \multicolumn{8}{|l|}{ Prior surgical resection } \\
\hline Present & 16.4 & 1.000 & & & 1.000 & & \\
\hline Absent & 11.6 & 1.718 & $1.151-2.662$ & 0.007 & 1.580 & $0.970-2.618$ & 0.19 \\
\hline \multicolumn{8}{|l|}{ Biliary drainage } \\
\hline Present & 12.2 & 1.000 & & & & & \\
\hline Absent & 13.2 & 1.044 & $0.756-1.450$ & 0.79 & & & \\
\hline White blood cells count & & 1.0001 & $1.00004-1.0001$ & 0.001 & 0.999 & $0.999-1.0001$ & 0.82 \\
\hline Hemoglobin & & 0.844 & $0.766-0.929$ & $<0.001$ & 1.037 & $0.908-1.185$ & 0.59 \\
\hline Platelets & & 0.990 & $0.970-1.009$ & 0.33 & & & \\
\hline Albumin & & 0.510 & $0.388-0.673$ & $<0.001$ & 0.642 & $0.401-1.020$ & 0.061 \\
\hline Total bilirubin & & 1.210 & $0.889-1.615$ & 0.22 & & & \\
\hline $\mathrm{LDH}$ & & 1.003 & $1.002-1.004$ & $<0.001$ & 1.002 & $1.0005-1.003$ & 0.006 \\
\hline ALP & & 1.0003 & $1.00004-1.00006$ & 0.027 & 1.0003 & $0.999-1.0006$ & 0.11 \\
\hline CRP & & 1.120 & $1.065-1.170$ & $<0.001$ & 1.009 & $0.923-1.092$ & 0.84 \\
\hline NLR & & 1.201 & $1.125-1.278$ & $<0.001$ & 1.117 & $1.019-1.219$ & 0.018 \\
\hline CEA & & 1.001 & $0.999-1.002$ & 0.13 & & & \\
\hline CA19-9 & & 1.00002 & $1.00001-1.00002$ & 0.007 & 1.00001 & $0.999-1.00002$ & 0.19 \\
\hline
\end{tabular}

$O S$ overall survival, $H R$ hazard ratio, $C I$ confidence interval, $L D H$ lactate dehydrogenase, $A L P$ alkaline phosphatase, CRP C-reactive protein, neutrophil-to-lymphocyte ratio, $C E A$ carcinoembryonic antigen, CA19-9 carbohydrate antigen 19-9, HR hazard ratio, $C I$ confidence interval ${ }^{\mathrm{a}} \mathrm{OS}$ was shown for only categorical variables

${ }^{\mathrm{b}}$ Bile duct; intrahepatic bile duct, extrahepatic bile duct and ampulla of Vater 

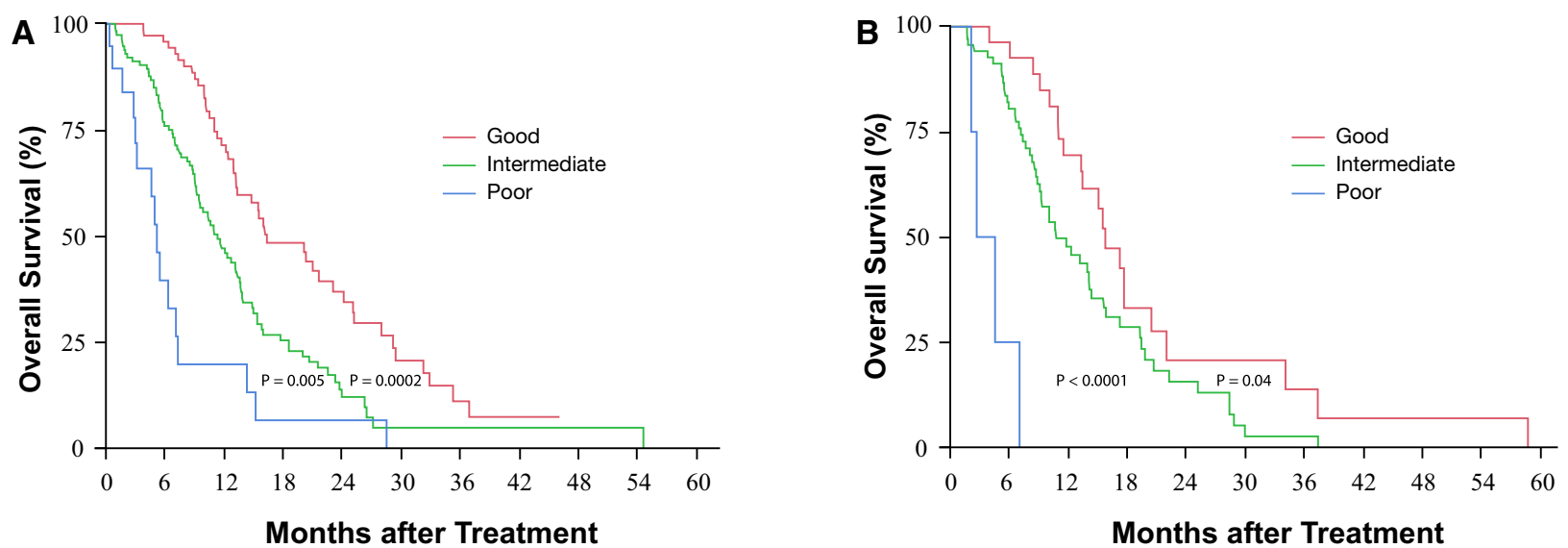

No. at Risk

$\begin{array}{rrrrrrrrrrrr}\text { Good } & 72 & 67 & 44 & 23 & 15 & 7 & 3 & 1 & 1 & 1 & 1 \\ \text { Intermediate } & 114 & 84 & 42 & 20 & 7 & 2 & 2 & 2 & 2 & 1 & 0 \\ \text { Poor } & 19 & 6 & 3 & 1 & 1 & 0 & 0 & 0 & 0 & 0 & 0\end{array}$

Fig. 2 Kaplan-Meier curves of overall survival according to the risk groups based on the number of poor prognostic factors in the investigation dataset (a) and validation dataset (b). The patients were divided into three risk groups as follows: (1) good-prognosis group, none of the poor prognostic factors present; (2) intermediateprognosis group, 1 or 2 poor prognostic factors present; (3) poorprognosis group, all poor prognostic factors present. The three poor

In conclusion, we identified three predictive factors of the OS in advanced BTC patients receiving GC therapy, which allowed these patients to be classified into three risk groups. These findings are expected to be helpful in decision-making on the first-line chemotherapy and survival estimation in patients with advanced BTC.

Acknowledgements The authors would like to thank Mr. Shogo Nomura for his valuable statistical advice and Ms. Kayo Takei for her great support.

\section{Compliance with ethical standards}

Conflict of interest Masafumi Ikeda has received honoraria from Bayer Yakuhin, Ltd., Taiho Pharmaceutical Co., Ltd., Novartis Pharma K.K., Bristol-Myers Squibb, and Eli Lilly Japan K.K., and received commercial research funding from Ono Pharmaceutical Co., Ltd., AstraZeneca K.K., Zeria Pharmaceutical Co., Ltd., Taiho Pharmaceutical Co., Ltd., Merck Serono, Bayer Yakuhin, Ltd., YakltHonsha Co., Ltd., Kyowa Hakko Kirin Co., Ltd., Eisai Co., Ltd., Eli Lilly Japan K.K., Baxter, ASLAN Pharmaceuticals, Nano Carrier Co., Ltd., and Chugai Pharmaceutical Co., Ltd.

Open Access This article is distributed under the terms of the Creative Commons Attribution 4.0 International License (http://crea tivecommons.org/licenses/by/4.0/), which permits unrestricted use, distribution, and reproduction in any medium, provided you give appropriate credit to the original author(s) and the source, provide a link to the Creative Commons license, and indicate if changes were made.

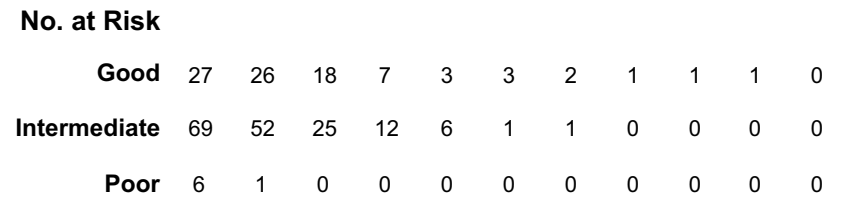

prognostic factors were elevated serum lactate dehydrogenase (cutoff value, $245 \mathrm{IU} / \mathrm{L}$; upper limit of normal), elevated neutrophil-tolymphocyte ratio (cutoff value, 3.0), and poor performance status (ECOG PS 0 vs. 1 or 2). There were statistically significant differences in the overall survival among the three groups in both the investigation and validation datasets. ECOG PS Eastern Cooperative Oncology Group Performance Status

\section{References}

1. Hori M, Matsuda T, Shibata A, et al. Cancer incidence and incidence rates in Japan in 2009: a study of 32 population-based cancer registries for the Monitoring of Cancer Incidence in Japan (MCIJ) project. Jpn J Clin Oncol. 2015;45:884-91.

2. Lepage C, Capocaccia R, Hackl M, et al. Survival in patients with primary liver cancer, gallbladder and extrahepatic biliary tract cancer and pancreatic cancer in Europe 1999-2007: results of EUROCARE-5. Eur J Cancer. 2015;51:2169-78.

3. Ishihara S, Horiguchi A, Miyakawa S, et al. Biliary tract cancer registry in Japan from 2008 to 2013. J Hepatobiliary Pancreat Sci. 2016;23:149-57.

4. Miyazaki Y, Kokudo T, Amikura K, et al. Survival of surgery for recurrent biliary tract cancer: a single-center experience and systematic review of literature. Jpn J Clin Oncol. 2017;47:206-12.

5. Valle J, Wasan H, Palmer DH, et al. Cisplatin plus gemcitabine versus gemcitabine for biliary tract cancer. $\mathrm{N}$ Engl $\mathrm{J}$ Med. 2010;362:1273-81.

6. Okusaka T, Nakachi K, Fukutomi A, et al. Gemcitabine alone or in combination with cisplatin in patients with biliary tract cancer: a comparative multicentre study in Japan. $\mathrm{Br} \mathrm{J}$ Cancer. 2010;103:469-74.

7. Mizusawa J, Morizane C, Okusaka T, et al. Randomized Phase III study of gemcitabine plus S-1 versus gemcitabine plus cisplatin in advanced biliary tract cancer: Japan Clinical Oncology Group Study (JCOG1113, FUGA-BT). Jpn J Clin Oncol. 2016;46:385-8.

8. Grenader T, Nash S, Plotkin Y, et al. Derived neutrophil lymphocyte ratio may predict benefit from cisplatin in the advanced 
biliary cancer: the ABC-02 and BT-22 studies. Ann Oncol. 2015;26:1910-6.

9. Bridgewater J, Lopes A, Wasan H, et al. Prognostic factors for progression-free and overall survival in advanced biliary tract cancer. Ann Oncol. 2016;27:134-40.

10. Kim BJ, Hyung J, Yoo C, et al. Prognostic factors in patients with advanced biliary tract cancer treated with first-line gemcitabine plus cisplatin: retrospective analysis of 740 patients. Cancer Chemother Pharmacol. 2017;80:209-15.

11. Agarwal R, Sendilnathan A, Siddiqi NI, et al. Advanced biliary tract cancer: clinical outcomes with $\mathrm{ABC}-02$ regimen and analysis of prognostic factors in a tertiary care center in the United States. J Gastrointest Oncol. 2016;7:996-1003.

12. Ishimoto U, Kondo S, Ohba A, et al. Prognostic factors for survival in patients with advanced intrahepatic cholangiocarcinoma treated with gemcitabine plus cisplatin as first-line treatment. Oncology. 2017;94:72-8.

13. Park HS, Park JS, Chun YJ, et al. Prognostic factors and scoring model for survival in metastatic biliary tract cancer. Cancer Res Treat. 2017;49:1127-39.

14. McNamara MG, Templeton AJ, Maganti M, et al. Neutrophil/lymphocyte ratio as a prognostic factor in biliary tract cancer. Eur J Cancer. 2014;50:1581-9.

15. Tang LQ, Hu DP, Chen QY, et al. Elevated high-sensitivity C-reactive protein levels predict decreased survival for nasopharyngeal carcinoma patients in the intensity-modulated radiotherapy era. PLoS One. 2015. https://doi.org/10.1371/jour nal.pone.0122965.

16. Park I, Lee JL, Ryu MH, et al. Prognostic factors and predictive model in patients with advanced biliary tract adenocarcinoma receiving first-line palliative chemotherapy. Cancer. 2009;115:4148-55.

17. Suzuki E, Furuse J, Ikeda M, et al. Treatment efficacy/safety and prognostic factors in patients with advanced biliary tract cancer receiving gemcitabine monotherapy: an analysis of 100 cases. Oncology. 2010;79:39-45.

18. Saisho T, Okusaka T, Ueno H, et al. Prognostic factors in patients with advanced biliary tract cancer receiving chemotherapy. Hepatogastroenterology. 2005;52:1654-8.

19. Furuse J, Okusaka T, Ohkawa S, et al. A phase II study of uraciltegafur plus doxorubicin and prognostic factors in patients with unresectable biliary tract cancer. Cancer Chemother Pharmacol. 2009;65:113-20.

20. Bridgewater J, Lopes A, Palmer D, et al. Quality of life, longterm survivors and long-term outcome from the ABC-02 study. Br J Cancer. 2016;114:965-71.

21. Doherty MK, McNamara MG, Aneja P, et al. Long term responders to palliative chemotherapy for advanced biliary tract cancer. J Gastrointest Oncol. 2017;8:352-60.

22. Masoud GN, Li W. HIF-1alpha pathway: role, regulation and intervention for cancer therapy. Acta Pharm Sin B. 2015;5:378-89.

23. Azuma M, Shi M, Danenberg KD, et al. Serum lactate dehydrogenase levels and glycolysis significantly correlate with tumor VEGFA and VEGFR expression in metastatic CRC patients. Pharmacogenomics. 2007;8:1705-13.

24. Faloppi L, Del Prete M, Casadei Gardini A, et al. The correlation between LDH serum levels and clinical outcome in advanced biliary tract cancer patients treated with first line chemotherapy. Sci Rep. 2016. https://doi.org/10.1038/srep24136.

25. Le A, Cooper CR, Gouw AM, et al. Inhibition of lactate dehydrogenase A induces oxidative stress and inhibits tumor progression. Proc Natl Acad Sci USA. 2010;107:2037-42.
26. Yu SL, Xu LT, Qi Q, et al. Serum lactate dehydrogenase predicts prognosis and correlates with systemic inflammatory response in patients with advanced pancreatic cancer after gemcitabine-based chemotherapy. Sci Rep. 2017. https://doi.org/10.1038/srep45194.

27. Zhou M, Zhao Y, Ding Y, et al. Warburg effect in chemosensitivity: targeting lactate dehydrogenase-A re-sensitizes taxol-resistant cancer cells to taxol. Mol Cancer. 2010. https://doi.org/10. 1186/1476-4598-9-33.

28. Fu J, Jiang $\mathrm{H}, \mathrm{Wu} \mathrm{C}$, et al. Overcoming cetuximab resistance in Ewing's sarcoma by inhibiting lactate dehydrogenase-A. Mol Med Rep. 2016;14:995-1001.

29. Giovannetti E, Leon LG, Gomez VE, et al. A specific inhibitor of lactate dehydrogenase overcame the resistance toward gemcitabine in hypoxic mesothelioma cells, and modulated the expression of the human equilibrative transporter-1. Nucleosides Nucleotides Nucleic Acids. 2016;35:643-51.

30. Faloppi L, Bianconi M, Giampieri R, et al. The value of lactate dehydrogenase serum levels as a prognostic and predictive factor for advanced pancreatic cancer patients receiving sorafenib. Oncotarget. 2015;6:35087-94.

31. Guthrie GJ, Charles KA, Roxburgh CS, et al. The systemic inflammation-based neutrophil-lymphocyte ratio: experience in patients with cancer. Crit Rev Oncol Hematol. 2013;88:218-30.

32. Templeton AJ, McNamara MG, Seruga B, et al. Prognostic role of neutrophil-to-lymphocyte ratio in solid tumors: a systematic review and meta-analysis. J Natl Cancer Inst. 2014. https://doi. org/10.1093/jnci/dju124.

33. Yao Y, Yuan D, Liu H, et al. Pretreatment neutrophil to lymphocyte ratio is associated with response to therapy and prognosis of advanced non-small cell lung cancer patients treated with firstline platinum-based chemotherapy. Cancer Immunol Immunother. 2013;62:471-9.

34. Keizman D, Ish-Shalom M, Huang P, et al. The association of pre-treatment neutrophil to lymphocyte ratio with response rate, progression free survival and overall survival of patients treated with sunitinib for metastatic renal cell carcinoma. Eur J Cancer. 2012;48:202-8.

35. Tang H, Lu W, Li B, et al. Prognostic significance of neutrophilto-lymphocyte ratio in biliary tract cancers: a systematic review and meta-analysis. Oncotarget. 2017;8:36857-68.

36. Cho KM, Park H, Oh DY, et al. Neutrophil-to-lymphocyte ratio, platelet-to-lymphocyte ratio, and their dynamic changes during chemotherapy is useful to predict a more accurate prognosis of advanced biliary tract cancer. Oncotarget. 2017;8:2329-41.

37. Lin EY, Pollard JW. Role of infiltrated leucocytes in tumour growth and spread. Br J Cancer. 2004;90:2053-8.

38. Coussens LM, Werb Z. Inflammation and cancer. Nature. $2002 ; 420: 860-7$

39. el-Hag A, Clark RA. Immunosuppression by activated human neutrophils. Dependence on the myeloperoxidase system. J Immunol. 1987;139:2406-13.

40. Loi S, Sirtaine N, Piette F, et al. Prognostic and predictive value of tumor-infiltrating lymphocytes in a phase III randomized adjuvant breast cancer trial in node-positive breast cancer comparing the addition of docetaxel to doxorubicin with doxorubicinbased chemotherapy: BIG 02-98. J Clin Oncol. 2013;31:860-7.

41. Gooden MJ, de Bock GH, Leffers N, et al. The prognostic influence of tumour-infiltrating lymphocytes in cancer: a systematic review with meta-analysis. Br J Cancer. 2011;105:93-103.

42. Li X, Dai D, Chen B, et al. The value of neutrophil-to-lymphocyte ratio for response and prognostic effect of neoadjuvant chemotherapy in solid tumors: a systematic review and metaanalysis. J Cancer. 2018;9:861-71. 